\title{
Detectors for fast X-ray timing and spectroscopy
}

\author{
Peter Lechner ${ }^{1}$ \\ PNSensor \\ Otto-Hahn-Ring 6, 81739 München, Germany \\ E-mail: peter.lechner@pnsensor.de
}

\section{Adrian Niculae}

PNDetector

Otto-Hahn-Ring 6, 81739 München, Germany

E-mail: adrian.niculae@pndetector.de

\section{Heike Soltau}

PNSensor

Otto-Hahn-Ring 6, 81739 München, Germany

E-mail: heike.soltau@pnsensor.de

\section{Lothar Strüder}

MPI für extraterrestrische Physik \& MPI Halbleiterlabor

Otto-Hahn-Ring 6, 81739 München, Germany

E-mail: lothar.strueder@hll.mpg.de

The High Time Resolution Spectrometer (HTRS) on board the International X-ray Observatory (IXO) is dedicated to the physics of matter at extreme density and gravity and will observe the $\mathrm{X}$-rays generated in the inner accretion flows around the most compact massive objects, i.e. black holes and neutron stars. As these are the brightest objects in the X-ray sky the HTRS has to work at extreme count rates and is therefore based on a Silicon Drift Detector (SDD). The SDD is an X-ray sensor combining a large sensitive area with a small output capacitance. It uses fast signal charge collection on an integrated amplifier by a focusing internal electrical field and is optimised for high resolution spectroscopy at high count rate. The HTRS sensor is a monolithic array of Silicon Drift Detectors (SDDs) with 31 cells in a circular envelope and a sensitive volume of $4.5 \mathrm{~cm}^{2} \times 450 \mu \mathrm{m}$. It is specified to provide energy spectra with a resolution of $150 \mathrm{eV}$ (FWHM at $6 \mathrm{keV}$ ) at high time resolution of $10 \mu \mathrm{sec}$ and with high count rate capability up to a goal of $2 \cdot 10^{6}$ counts per second, corresponding to a 12 Crab equivalent source. As the HTRS is a non-imaging instrument and will target only point sources it is placed on axis but out of focus so that the spot is spread over the array of 31 SDD cells.

Fast X-ray timing and spectroscopy at extreme count rates: Science with the HTRS on the International $X$-ray Observatory -HTRS 2011

Champéry, Switzerland

February 7-11 2011

\footnotetext{
$1 \quad$ Speaker
} 


\section{Introduction}

The International X-ray Observatory (IXO) [1] is a joint high-energy mission of the USAmerican, European, and Japanese space agencies with a projected launch in 2021. To address some of the fundamental questions in contemporary astrophysics and cosmology (matter under extreme conditions, formation and evolution of galaxies, clusters and large scale structure, life cycles of matter and energy) IXO requires high sensitivity and high spatial resolution over a broad energy band with excellent energy and time resolution. Therefore, IXO combines a large aperture X-ray mirror (collecting area $3 \mathrm{~m}^{2}$, focal length $20 \mathrm{~m}$, angular resolution 5 arcsec) with a set of six specialized complementary sensor systems focal plane sensors.

The High Time Resolution Spectrometer (HTRS) [2] is the only focal plane instrument matching the IXO top level requirement to observe a bright X-ray source of the intensity of one Crab with less than $10 \%$ dead time. The HTRS science will focus on matter under extreme conditions, by simultaneous fast X-ray timing and high-resolution spectroscopy measurements of compact objects (black holes, neutron stars) powered by accretion. As the sources to be observed by HTRS are the brightest in the X-ray sky the instrument design is driven by the capability to operate at extremely high count rates. The only available sensor concept that is able to cope with the HTRS requirements summarized in table 1 is the Silicon Drift Detector (SDD). It combines a large sensitive area with an extremely small readout capacitance and is therefore predetermined for high-rate high-resolution X-ray spectroscopy. The HTRS instrument is based on a monolithic array of 31 SDD cells. As HTRS is a non-imaging instrument and will target only point sources it is placed on axis but out of focus so that the intense photon flux is spread evenly over the array of 31 SDD cells.

\begin{tabular}{|l|l|l|}
\hline energy range & $0.3-15 \mathrm{keV}$ & \\
\hline time resolution & $10 \mu \mathrm{sec}$ & \\
\hline energy resolution $\left(-40^{\circ} \mathrm{C}\right)$ & $150 \mathrm{eV}$ & goal at beginning of lifetime \\
\hline FWHM @ $5.9 \mathrm{keV}$ & $\leq 200 \mathrm{eV}$ & requirement at beginning of lifetime \\
\hline & $\leq 300 \mathrm{eV}$ & requirement at end of lifetime \\
\hline count rate capability & $>10 \mathrm{Crab}$ & $1 \mathrm{Crab} \approx 170.000$ photons $/ \mathrm{sec}$ \\
\hline deadtime \& pile-up & $<2 \% @ 1 \mathrm{Crab}$ & \\
\hline
\end{tabular}

Table 1. The sensor requirements of the IXO High Time Resolution Spectrometer

\section{Silicon Drift Detector}

A Silicon Drift Detectors (SDD) is a large volume of a high resistivity semiconductor, in our case $n$-type silicon, which is depleted by a small sized $n^{+}$-bulk contact reverse biased with respect to $\mathrm{p}^{+}$-junctions covering both surfaces of the structure. The $\mathrm{p}^{+}$-junctions are strip-like segmented and biased in such a way that an electric field parallel to the surface exists [3]. Electrons released within the depleted volume by the absorption of ionizing radiation or by thermal generation drift towards the $\mathrm{n}^{+}$-substrate contact, which acts as collecting anode and is connected to an amplifier. The generated holes are drained away by the $\mathrm{p}^{+}$-junctions. 
In an advanced SDD design optimized for applications in photon spectroscopy the $\mathrm{p}^{+}$-strip system for the generation of the drift field has the shape of concentric rings. The drift rings and the collecting anode in their centre are placed on one side of the structure, while the opposite surface is covered by a non-structured $\mathrm{p}^{+}$-junction acting as homogeneous, thin radiation entrance window [4,5] (fig. 1). There is no field-free region in the device, and the whole volume is sensitive for the absorption of ionizing radiation. The typical signal charge drift time is of the order of $100 \mathrm{nsec}$. SDDs of this type do not provide position information but are used as energy dispersive spectrometers, taking advantage of the small value of the anode capacitance, guaranteeing high count rate capability and excellent, CCD-like energy resolution.
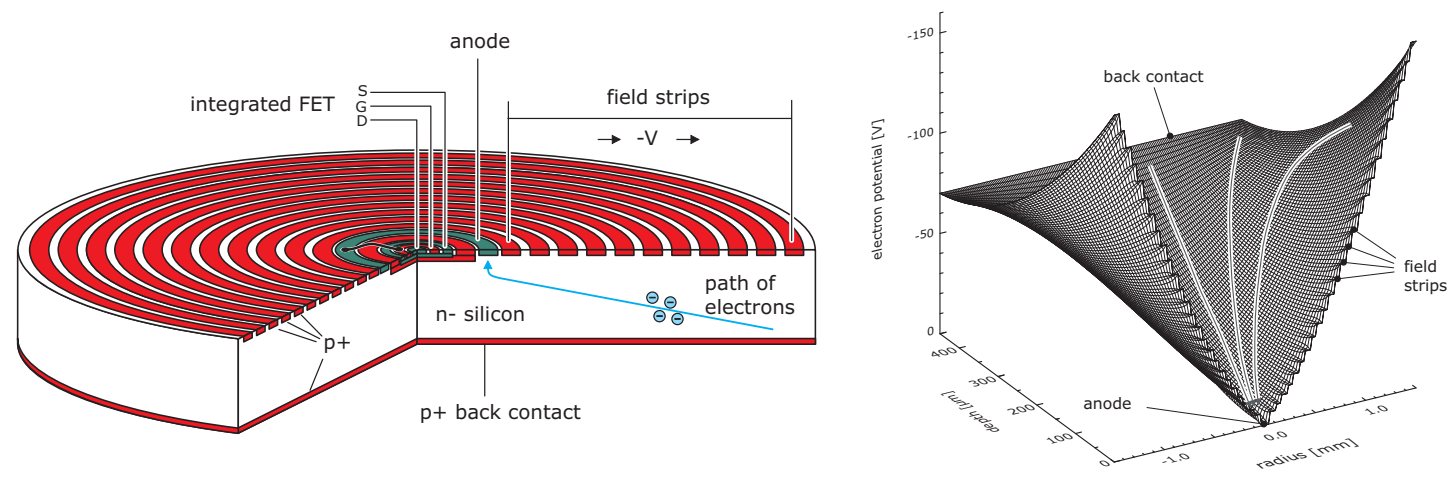

Figure 1. Schematic view of a cylindrical Silicon Drift Detector and its calculated potential distribution.

An additional gain in high-rate performance and energy resolution is achieved by integrating the first transistor of the amplifying electronics on the SDD chip [6]. That way the SDD also gets robust with respect to microphonic noise and electrical pickup. Due to its minimized overall capacitance in the order of $100 \mathrm{fF}$ the SDD with integrated transistor has its optimum noise figure at extremely short shaping time constants $\leq 1 \mu \mathrm{sec}$ and it works at photon rates exceeding $10^{5} \mathrm{cps}$ (counts per second) without degradation in spectroscopic performance. The low leakage current level obtained by the elaborated process technology allows operating SDDs at room temperature or with moderate cooling, e.g. by a thermoelectric cooler.

SDDs of this type have been produced in large numbers and are available in a multitude of shapes and sizes from $5 \mathrm{~mm}^{2}$ up to $1 \mathrm{~cm}^{2}$. They are primarily used for X-ray spectroscopy in industrial instrumentation and scientific experiments. SDDs have also gained space experience in the Alpha Particle X-ray Spectrometers on the robotic arms of the NASA Mars Exploration Rovers Spirit and Opportunity [7]. To increase the sensitive area without loss in performance monolithic SDD arrays have been developed [8].

\section{SDD for the High Time Resolution Spectrometer}

\subsection{Layout}

The HTRS sensor is a multi-channel SDD consisting of 31 cells in a circular envelope of $24 \mathrm{~mm}$ diameter and a total area of $4.5 \mathrm{~cm}^{2}$. The central cell has a circular shape. The outer cells have the shape of ring sectors and are arranged in three rings of $1 \times 6$ and $2 \times 12$ cells (fig. 2). 
All cells have equal areas of $14.6 \mathrm{~mm}^{2}$. The thickness of the Silicon wafer is $450 \mu \mathrm{m}$. The 31 SDD cells are logically grouped in four 'quadrants' of seven or eight cells. The quadrants are to a large extent electrically independent, including redundant supply and readout electronics. The readout structures of each quadrant's cells are connected to an eight-channel readout chip.

The border lines between the cells' entrance windows is masked by a baffle to inhibit 'split events' caused by photons absorbed along the cell borders. Split events generate signal charges in two or more neighbouring cells and cause false energy information. The baffle has $200 \mu \mathrm{m}$ wide spokes and covers a $10 \%$ fraction of the total sensitive area, but it increases the spectral quality as the sensor cells will receive the full signal of single events.
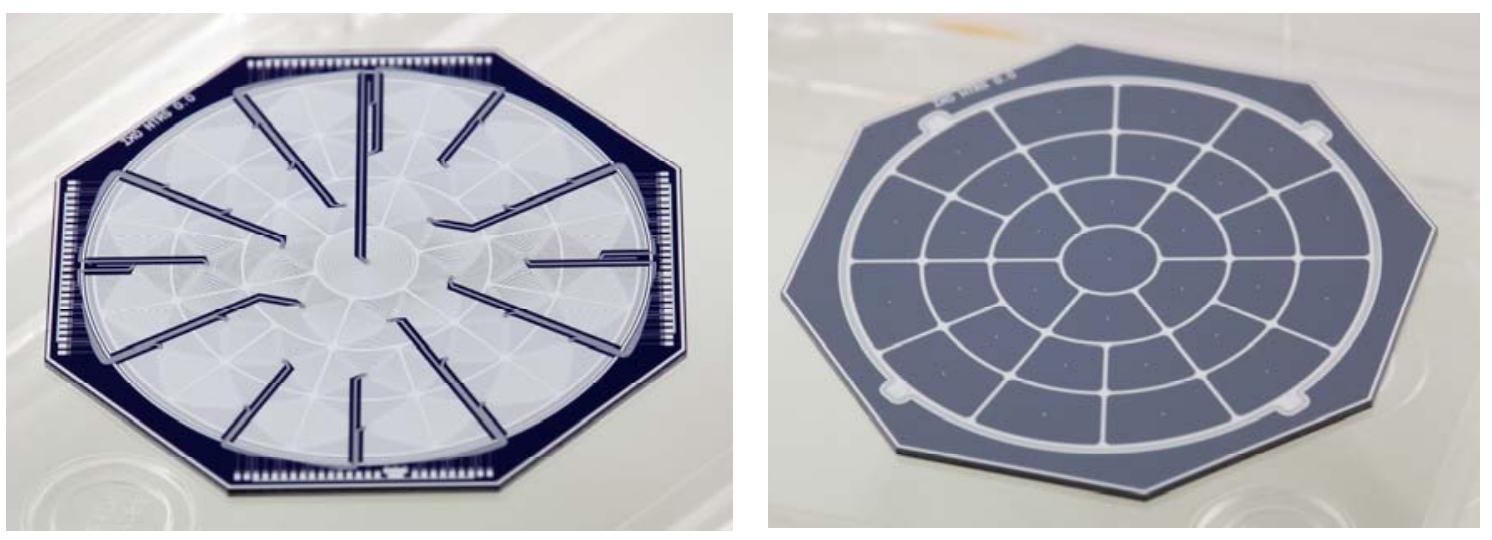

Figure 2. Mechanical sample of the HTRS sensor, readout side (left) and radiation entrance window side (right).

\subsection{Performance}

At the HTRS conditions, i.e. sensor temperature $-40{ }^{\circ} \mathrm{C}$ and short signal processing time for high count rate handling, the SDD energy resolution will be $\leq 150 \mathrm{eV}$ FWHM at $5.9 \mathrm{keV}$ at the beginning of the lifetime. The energy resolution is stable within $2 \%$ and the gain variation is less than $2 \%$ even at count rates $>10^{5}$ photons per second and SDD cell, corresponding to a source intensity $>10 \mathrm{Crab}$ (fig. 3). The SDD's quantum efficiency is above $90 \%$ in the energy band from 0.5 to $10 \mathrm{keV}$. Photons at higher energies are increasingly transmitted through the bulk thickness of $450 \mu \mathrm{m}$ without interaction. At low energies the quantum efficiency is limited by the entrance window's Si dead layer and by a thin Al layer deposited on the window.

SDDs processed in the HTRS-like technology have proven to be radiation hard with respect to X-rays up to $10^{13}$ absorbed photons (Mo-K, $18 \mathrm{keV}$ ) without degradation of the energy resolution. This figure would theoretically allow for a ten years' continuous observation of a bright source with an intensity of several Crabs. In addition to photon irradiation the HTRS sensor will be exposed to $2 \cdot 10^{9}\left(5 \cdot 10^{9}\right)$ protons (10 MeV equivalent) per $\mathrm{cm}^{2}$ in a 5 (10) years mission, assuming an effective shielding represented by $15 \mathrm{~mm}$ of Aluminium. Protons create defects in the bulk, which are generation centres for leakage current. Based on experimental data [9] this non-ionizing radiation effect has been modelled to cause an additional leakage current of $2.43 \cdot 10^{-17} \mathrm{~A}$ at room temperature per $10 \mathrm{MeV}$ proton. The corresponding deterioration of the energy resolution is $\leq 250 \mathrm{eV}$ FHWM at $5.9 \mathrm{keV}$ towards the end of the mission lifetime, still meeting the system specifications within a sufficient margin. 


\subsection{Readout}

The first FET integrated on the SDD chip is configured as a charge sensitive amplifier (CSA, fig. 3) [10]. For the capacitive feedback the parasitic capacitance of the reverse biased junction between the charge collecting anode and a guard ring is used, its value is about $30 \ldots 35 \mathrm{fF}$. The leakage current and the signals are integrated on this feedback capacitance $\mathrm{C}_{\mathrm{fb}}$ generating a voltage ramp with superposed steps at the pre-amplifier output. Whenever the output voltage exceeds a preset threshold value the readout chip produces a signal which is used to trigger an external reset circuit discharging the SDD. For this purpose a $\mathrm{p}^{+}$-doped reset diode is integrated directly in the anode. The reset diode is reverse biased $(-15 \mathrm{~V})$ during signal integration and pulsed to forward bias $(+7 \mathrm{~V})$ for the reset with a pulse duration of $300 \ldots 500 \mathrm{nsec}$. The reset signal is common for all channels of a quadrant, and so the average reset period is determined by the channel with the highest sum of leakage and signal currents.

The readout configuration with CSA and pulsed reset has been tested using a $5 \mathrm{~mm}^{2} \mathrm{SDD}$ and conventional discrete components for the electronic chain. The spectroscopy performance is almost independent of the input count rate up to $10^{5} \mathrm{cps}$ : the energy resolution (FWHM at $5.9 \mathrm{keV}$ ) is stable on the $1 \%$ level and the peak position is stable on the $0.1 \%$ level (fig. 3 ).
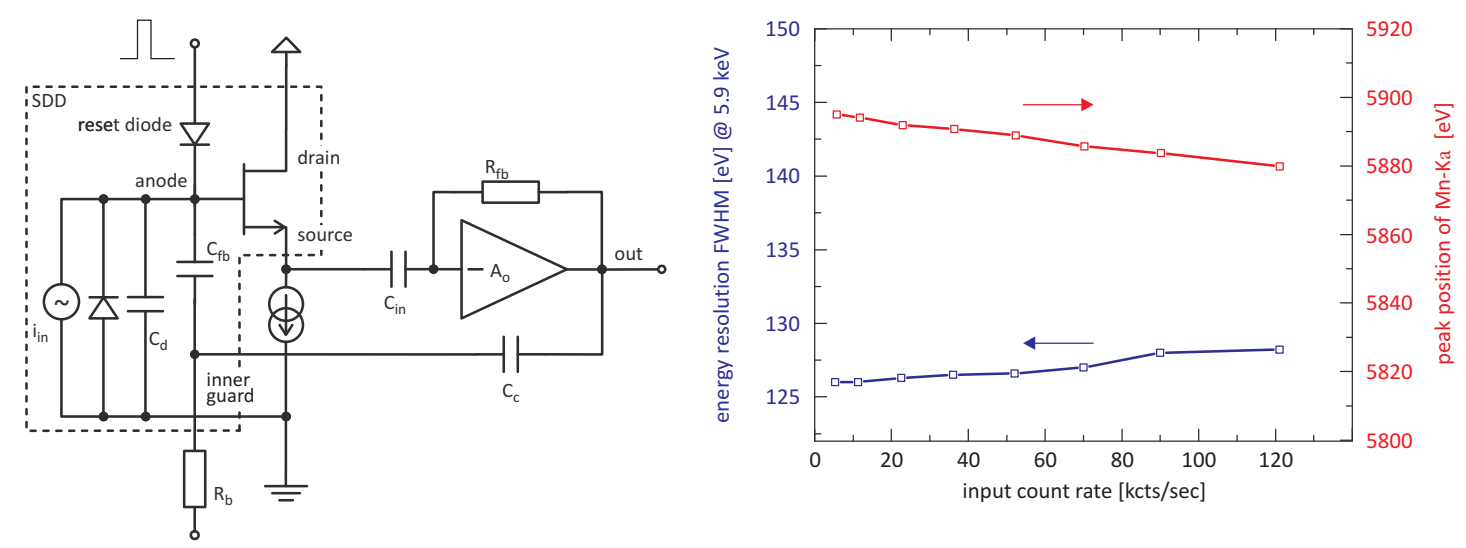

Figure 3. Charge sensitive amplifier configuration of the integrated FET (left) and measured high rate performance of a $5 \mathrm{~mm}^{2} \mathrm{SDD}$ (right).

The baseline implementation of the readout electronics is an analog chain integrated in an 8-channel ASIC. It includes a pre-amplifier, a filter with $600 \mathrm{nsec}$ peaking time, a baseline holder, an event trigger, a peak stretcher, and a fast (200 nsec) shaper channel for pile-up detection and rejection. The analog amplitudes of the eight channels and the channel addresses are delivered to an external ADC by an 8/1 analog multiplexer operating at $10 \mathrm{MHz}$.

As an alternative a digital readout chain is under study. In the digital option the configuration and operation mode of the integrated first FET is unchanged. The readout chip includes eight pre-amplifiers only. The pre-amplifier output signals of each channel are sampled by a fast $(150 \mathrm{MHz}) 14 \mathrm{bit} \mathrm{ADC}$ and filtered by software filter functions with effective time constants comparable to the analog shaper. The digital option is of great flexibility, as its function is mainly based on software which can be developed and adapted even after the launch. 


\subsection{Focal Plane}

As HTRS is a non-imaging instrument operating at high count rates the sensor is displaced from the focal plane to distribute the photon flux over the 31 SDD cells. With the actual mirror design $\left(3 \mathrm{~m}^{2}\right.$ at $\left.1.25 \mathrm{keV}\right)$ the out-of-focus distance is $11.3 \mathrm{~cm}$. The out-of-focus distance is chosen such that with the given SDD format $99.9 \%$ of the incoming photons fall into the sensor area even in the worst case misalignment scenario. The thermal, mechanical and electrical interface to the HTRS sensor is given by the sensor hybrid. To keep the quadrant philosophy an individual hybrid is assigned to each sensor quadrant. Per quadrant there is one power flex-lead connected to the electronics box and carrying the sensor supply voltages and one signal flexlead connected to the readout board and including the signal, feedback and trigger lines. To keep the setup compact the active components of the readout electronics are placed on a readout board separated from the sensor by a graded shield plate. Both the sensor hybrid and the readout board are enclosed by an Al box acting both as radiation shield and thermal interface (fig. 4).
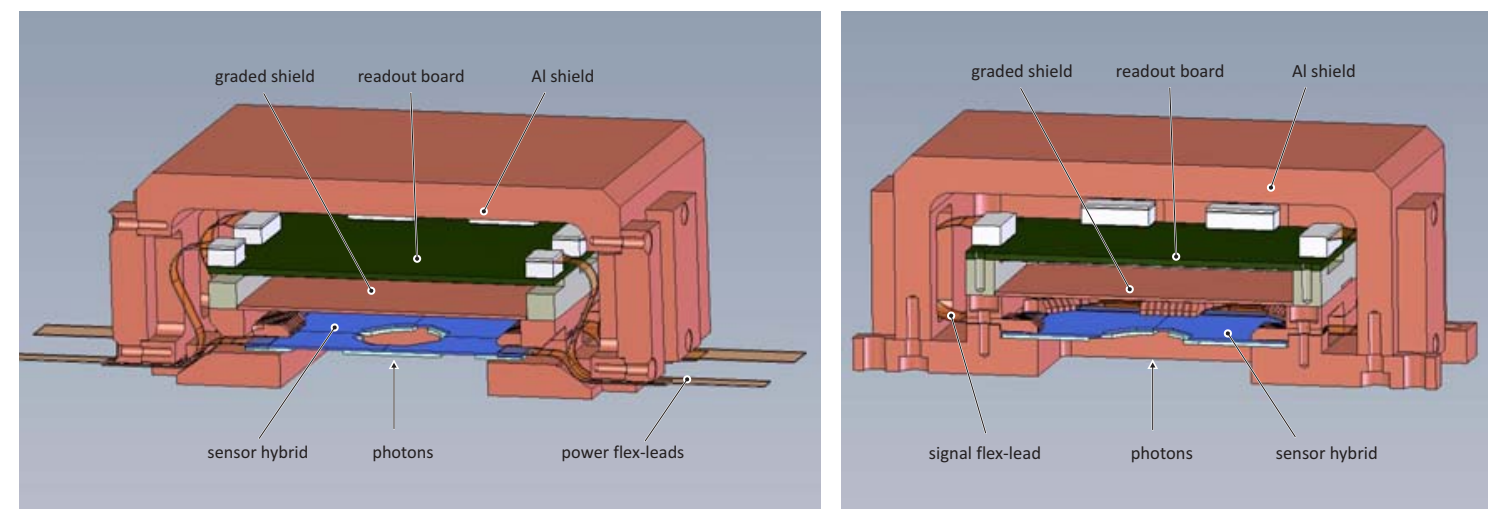

Figure 4. Two perpendicular cross sections of the HTRS sensor assembly.

\section{References}

[1] http://ixo.gsfc.nasa.gov

[2] Barret D. et al., The High Time Resolution Spectrometer (HTRS) aboard the International X-ray Observatory (IXO), Proc. SPIE 7732 (2010) 77321M

[3] Gatti E., Rehak P., Semiconductor drift chamber - An application of a novel charge transport scheme, Nucl. Instr. and Meth. A 225 (1984) 608-614

[4] Kemmer J., Lutz G., New detector concepts, Nucl. Instr. and Meth. A 253 (1987) 365-377

[5] Lechner P. et al., Silicon drift detectors for high resolution room temperature X-ray spectroscopy, Nucl. Instr. and Meth. A 377 (1996) 346-351

[6] Pinotti E. et al., The pn-CCD on-chip electronics, Nucl. Instr. and Meth. A 326 (1993) 85-91

[7] Rieder R. et al., The new Athena alpha particle X-ray spectrometer for the Mars Exploration Rovers, Journal of Geophysical Research E: Planets 108 (12) (2003) ROV7-1-ROV7-13

[8] Lechner P. et al., Multichannel silicon drift detectors for X-ray spectroscopy, Proc. SPIE 4012 (2000) 592-599

[9] Segneri G. et al., Measurement of the current related damage rate at-50 ${ }^{\circ} \mathrm{C}$ and consequences on macropixel detector operation in space experiments, IEEE TNS 56(6) (2009) 3734-3742

[10] Niculae A. et al., Optimized readout methods of silicon drift detectors for high-resolution X-ray spectroscopy, Nucl. Instr. and Meth. A 568 (2006) 336-342 